\title{
50 Jahre Gesellschaft für Pädiatrische Radiologie - eine Herausforderung
}

\author{
50 Years Gesellschaft für Pädiatrische Radiologie - A Challenge
}

G. Benz-Bohm, B. Stöver, M. Riccabona

\section{Bibliografie}

Dol http://dx.doi.org/10.1055/ s-0033-1350205

Fortschr Röntgenstr 2013; 185: 807-809 @ Georg Thieme Verlag KG Stuttgart · New York ISSN 1438-9029

\section{Korrespondenzadresse}

Prof. Dr. med. G. Benz-Bohm

Birresborner Str. 40

50935 Köln

g.benz-bohm@t-online.de

\section{Univ. Prof. Dr. med. Brigitte Stöver Im Sonnenschein 62 42719 Solingen stoevb@t-online.de}

Univ. Prof. Univ. Doz. Dr. med. Michael Riccabona

Univ. Klinikum LKH Graz, Österreich michael.riccabona@klinikumgraz.at

\section{Chronik}

$\nabla$

Bereits 1897 wurde im Anna-Kinderspital in Graz unter dem ersten Ordinarius für Kinderheilkunde der Medizinischen Fakultät, Theodor Escherich, die erste Röntgeneinrichtung in einem Kinderspital in Europa installiert. Erste Publikationen und Monografien über Röntgendiagnostik und -therapie im Kindesalter gehen in den deutschsprachigen Ländern auf Pädiater zurück [1, 2]. So entwickelte sich dort die Pädiatrische Radiologie aus der Pädiatrie und wurde erst später infolge der rasanten technischen Entwicklung der Radiologie angegliedert - oft als kleine Einheit mit geringer Ausstattung. Die Pädiatrischen Radiologen waren und sind zumeist „Einzelkämpfer“. In den USA, in Frankreich und England hingegen entwickelte sich die Pädiatrische Radiologie aus der Radiologie mit schon bald eigenständigen Instituten in größeren Pädiatrischen Zentren.

Am 17.9.1963 gründeten M. A. Lassrich (Mitbegründer der European Society of Pediatric Radiology, ebenfalls 1963), E. Willich, K.-D. Ebel und F. Schmid die Arbeitsgemeinschaft für Pädiatrische Radiologie in Köln und bildeten auch ihren ersten Vorstand. Ziel war zunächst der Zusammenschluss aller hauptamtlich tätigen Kinderradiologen und röntgenologisch tätigen Pädiatern mit jährlichen Sitzungen im Rahmen der Jahrestagung der Deutschen Gesellschaft für Kinderheilkunde. Die offizielle Gründung der AG für Pädiatrische Radiologie als e.V. fand am 6.6.1968 in Bonn statt - mit zu diesem Zeitpunkt 48 Mitgliedern und fortan eigenständigen Jahrestagungen. Diese wurde auf der Jahrestagung 1970 in Zürich zur Gesellschaft für Pädiatrische Radiologie e.V. für die Kinderradiologen des gesamten deutschen Sprachraums. Als offizielle Zeitschrift wurde Pediatric Radiology, Springer Verlag, 1973 eingeführt. 1990 erfolgte der Zusammenschluss der Kinderradiologen der neuen Bundesländer mit der GPR.

Auf dem Kongress der Deutschen Röntgengesellschaft 1965 in Nürnberg war Pädiatrische Radiologie erstmals Hauptthema. Zur Anlehnung an die DRG kam es 1969 durch Bildung einer Sektion. Die Anerkennung der Pädiatrischen Radiologie als Subspezialität der Radiologie erfolgte in Deutschland 1987, in der Schweiz 1990 und in Österreich bislang noch nicht [3].

\section{Jahre danach \\ $\nabla$}

Krankenversorgung

Nach den letzten verfügbaren Angaben des Statistischen Bundesamtes Wiesbaden vom 31.12.2010 sind in Deutschland 112 Radiologen mit Schwerpunkt Kinderradiologie berufstätig. Das sind weniger als 1,6\% der diagnostisch tätigen Radiologen, da nicht alle auch kinderradiologisch tätig sind. Bezogen auf die Anzahl der Kinder und Jugendlichen bis zum 18. Lebensjahr von über 13 Millionen, entsprechend 16\% der Gesamtbevölkerung, ist die Anzahl damit verschwindend gering [4]. Das bedeutet, die meisten Kinder und Jugendlichen werden von Radiologen untersucht, die keine spezielle Ausbildung haben. Im Gegensatz zu Schweiz und Österreich sind Kenntnisse in Kinderradiologie zur Facharztprüfung nicht in allen Bundesländern erforderlich. Entsprechend dem Intensivkurs in Kinderradiologie zur Vorbereitung auf die Facharztprüfung in Österreich gibt es jedoch seit Ende 2009 auch kinderradiologische Fortbildungen im Rahmen der „Fit für den Facharzt“-Serie der DRG (und der OERG).

Von großem Vorteil wäre, wenn Radiologen gegen Ende ihrer Facharztausbildung 3-6 Monate in die Kinderradiologie von Universitätskliniken oder größeren Kinderkliniken wechseln würden. Die Kinderradiologie käme aus ihrer Isolation und hätte einen radiologisch weitgehend ausgebildeten Kollegen vor Ort. Dieser würde in die altersentsprechenden Besonderheiten der Physiologie, Anatomie und Pathologie des Kindes und Jugendlichen eingeführt, den speziellen Strahlenschutz erlernen und damit auch die Kriterien für Wahl und Einsatz der Verfahren, und er würde in Sonografie und Nativdiagnostik ausgebildet. Darüber hinaus böte sich die Möglichkeit gemeinsamer wissenschaftlicher Tätigkeit.

Eine Mindestweiterbildung in Kinderradiologie mit kontinuierlicher Fortbildung für die Radiologen, die auch Kinder und Jugendliche betreuen, wäre ein Schritt in Richtung auf eine flächendeckende Grundversorgung. Darüber wird derzeit auch in der DRG im Rahmen der geplanten Weiterbildungsreform, ebenso in der OERG und auch auf europäischer Ebene diskutiert.

\section{Forschung und Lehre}

Forschung von heute bestimmt die Kinderradiologie von morgen. Mangelnde Forschung bedeutet für die Zukunft einen Qualitätsverlust in der studentischen Lehre, in der kinderradiologischen Aus- und Weiterbildung und in der radiologischen Versorgung der Kinder und Jugendlichen, und damit einen Qualitätsverlust für den Schwerpunkt Kinderradiologie.

Eigenständige Forschung und Lehre sind für die Berechtigung universitärer Abteilungen ver- 
pflichtend und sind für die Kinderradiologie ebenso zu fordern, wie sie für die Radiologie selbstverständlich sind.

Deutschland verfügt über 36 Universitätskliniken. 12 Leiter universitärer Kinderradiologien sind habilitiert und 2 von Kinderradiologischen Abteilungen akademischer Lehrkrankenhäuser. Österreich verfügt über 4 Universitätskliniken. Der Leiter und 2 weitere Kinderradiologen der einzigen universitären kinderradiologischen Abteilung sind habilitiert, im weiteren 2 Kinderradiologinnen einer anderen Universitätsklinik und ein niedergelassener Kinderradiologe. In der Schweiz sind es 5 Universitätskliniken mit 3 habilitierten Kinderradiologen in leitender Position und ein Leiter der Kinderradiologischen Abteilung eines Krankenhauses. Für Deutschland konnte ermittelt werden, dass trotz abnehmender universitärer Präsenz aktuelle Themen wissenschaftlich adäquat bearbeitet werden [5]. Gleiches gilt für Österreich und die Schweiz.

Dauerhaft jedoch kann nur eine bedarfsgerechte Anzahl universitärer kinderradiologischer Abteilungen kinderradiologische Basisausbildung gewährleisten und Spezialisierungsstellen sowie Forschung anbieten.

Zukunftsorientierte Forschung in der Kinderradiologie ist nur möglich mithilfe z.B. einer Radiologischen Universitätsklinik, der Infrastruktur und der Ressourcen eines Max-Planck-Instituts oder eines Deutschen Krebsforschungszentrums.

Ist vernetzte Forschung das Modell der Zukunft? Für die Kinderradiologie ist sie von Bedeutung, da sie eine wesentliche wissenschaftliche Basis schafft durch die Möglichkeit klinischer Forschung mittels multizentrischer Studien. Für die ESPR hat Prof. Fotter, Graz, das „European Excellence Network on Pediatric Radiology Research“ initiiert mit der Möglichkeit der Forschungsförderung durch die Europäische Union.

Teleradiologie in der Kinderradiologie ist wichtig für die weltweite wissenschaftliche Kommunikation, ebenso als ergänzende Lehrmöglichkeit. Im Rahmen der Patientenversorgung und der Basisausbildung hingegen kann sie den in der Kinderradiologie erforderlichen unabdingbaren Patientenkontakt und die praktische Unterweisung (z. B. Sonografie) nicht ersetzen.

\section{Effektivität und Wirtschaftlichkeit}

Die Pädiatrische Radiologie umfasst die gesamte Entwicklungsund Wachstumsphase, pränatal beginnend bis hin zum Adoleszentenalter. Aufgrund verbesserter Therapie und Diagnostik müssen auch zunehmend erwachsene Patienten mit früher rein pädiatrischen Erkrankungen in den kinderradiologischen Zentren weiterversorgt werden (z. B. kongenitale Vitien, zystische Fibrose).

Die Effektivität der pädiatrisch radiologischen Diagnostik liegt im frühen Erkennen eines Krankheitsbilds, das durch korrekt gewählte bildgebende Verfahren diagnostiziert wird. Die daraus folgende rechtzeitige Therapie und der Heilerfolg haben nicht nur Auswirkungen auf das Wohl des Kindes, sondern auch auf das Allgemeinwohl.

Drei Kriterien bestimmen somit die Untersuchungen in der Pädiatrischen Radiologie:

- Strahlenschutz - ALARA - as low as reasonably achievable

- Effektivität - Auswirkung auf Diagnose, Therapiewahl und Heilerfolg

- Effizienz - Auswahl aus den verschiedenen Verfahren unterschiedlicher Leistungsfähigkeit

\section{Strahlenschutz}

Die Rechtfertigung und Optimierung einer bildgebenden diagnostischen Untersuchung liegt in der Verantwortung dessen, der sie durchführt. Diese Aufgabe setzt nicht nur eine Fach-, sondern auch eine klinische Sachkunde voraus.

Nach Angaben des International Committee of Radiation Protection (ICRP 60/1990) geht eine Strahlenexposition bei Kindern unter 10 Jahren mit einem 3- bis 4-fach höheren Lebenszeitrisiko einher, verglichen mit dem 30.-40. Lebensjahr, und trägt ein größeres Risiko, einer Krebserkrankung in höherem Lebensalter zu erliegen [6].

Bereits 1996 hat die ENPR (European Network of Pediatric Radiology, ehem. Lake Starnberg Group) Europäische Richtlinien veröffentlicht [7]. 2010 wurden die Referenzwerte für diagnostische und interventionelle Röntgenuntersuchungen vom Bundesamt für Strahlenschutz aktualisiert [8].

Sonografie und MRT haben die diagnostischen Strategien in der Kinderradiologie verändert und dadurch eine Senkung der medizinisch verursachten Strahlenexposition bei Kindern und Jugendlichen erreicht. Die zunehmende strukturelle und personelle Reduzierung der Kinderradiologie läuft Gefahr, das Erreichte zunichte zu machen.

\section{Effektivität und Effizienz}

Da chronische Erkrankungen von Kindern länger erlebt werden, muss der Einsatz von Untersuchungen mit ionisierenden Strahlen besonders streng indiziert sein.

Die Prognose onkologischer Erkrankungen ist vom korrekten Einsatz der Bildgebung abhängig. Noch immer haben Kinder mit Knochen- oder Weichteiltumoren zum Teil eine lange, über Monate reichende Anamnese, obwohl die initiale Bildgebung den Tumor bereits darstellte. Therapeutische Konsequenzen und damit der Verlauf der Erkrankung werden wesentlich von der Bildgebung bestimmt, die dadurch auch zur hohen Heilungsrate von über $70 \%$ der kindlichen Tumorerkrankungen beiträgt. Ein häufiger Einsatz radiologischer Diagnostik kann jedoch eine Induktion von Zweittumoren bewirken.

Als Tumor verkannte Normvarianten bedingen unnötige Untersuchungen - abgesehen von der psychischen Belastung.

Pränatal erkannte und postnatal bestätigte Fehlbildungen können korrigiert werden, bevor Symptome oder Komplikationen auftreten. Auch die angeborene Hüftdysplasie wird durch das sonografische Hüftscreening spätestens in der 6. Lebenswoche diagnostiziert, sodass operative Korrekturen nur noch selten erforderlich sind.

Bei Früherkennen metabolischer Erkrankungen ist die Bildgebung zur Heilung oder lebenslanger Therapie entscheidend.

Das Erkennen typischer Skelettveränderungen nach Traumen als nicht akzidentell im Sinne einer Kindesmisshandlung ist von existenzieller Bedeutung für das gefährdete Kind.

Der Schutz der Kinderradiologie vor ökonomischen Zwängen muss auch in Zeiten knapper Ressourcen gewährt sein, um Kinderradiologische Diagnostik mit allen bildgebenden Verfahren effektiv betreiben zu können: Einerseits erforderliche radiologische Untersuchungen mit der größtmöglichen Reduktion der Strahlenexposition durchzuführen [9], andererseits durch strenge Handhabung der rechtfertigenden Indikation insbesondere bei sehr jungen Patienten das Lebenszeitrisiko ebenso wie die Möglichkeit einer Krebserkrankung in höherem Lebensalter bereits zum Zeitpunkt einer bildgebenden Diagnostik zu bedenken und entsprechend der Fragestellung das richtige Verfahren einzusetzen [10]. Durch ein zutreffendes Ergebnis erübrigen sich dann weitere Untersuchungen und reduzieren sich mögliche Spätfolgen einer rechtzeitig erkannten Erkrankung. Trotz Kostenintensität ist dies ein ökonomischer Gewinn. 
Das an Diagnosen geknüpfte Fallpauschalensystem im Gesundheitswesen in Deutschland, G-DRG-System, spiegelt die tatsächlichen kinderradiologischen Leistungen nicht wider, da diese nur zu einem geringen Teil erfasst und auch in ihrem tatsächlichen Aufwand unterschätzt werden. Dies ist erneut zu diskutieren und bedarf dringlichster Änderung.

Die Pädiatrische Radiologie von heute trägt Verantwortung für die Gesellschaft von morgen. Dies ist eine Herausforderung an die Radiologie. Auch in diesem Sinne ist Radiologie Zukunft.

\section{Literatur}

1 Feilchenfeld . Röntgenaufnahme von Spina ventosa an den Phalangen. Berlin: Klin Wschr 1896; 33: 403

2 Reyher P. Das Röntgenverfahren in der Kinderheilkunde. In: Bauer $\mathrm{H}$ (Hrsg). Bibliothek der physikalisch-medizinischen Techniken Bd. 4. Berlin: Hermann Meusser; 1912

3 Benz-Bohm G, Richter E. Chronik der Kinderradiologie. Berlin Heidelberg: Springer; 2012
4 Statistisches Bundesamt Wiesbaden. Bevölkerung nach Altersjahren. Ärztinnen/Ärzte nach Bezeichnung und ärztlicher Tätigkeit. Stichtag 31.12.2010. http://www.destatis.de

5 Hirsch W, Sorge I. Ein Literatur-Review zur wissenschaftlichen Präsenz der deutschen Kinderradiologie in Publikationen der Jahre 2009-2011. Fortschr Röntgenstr 2011; 183: 1012 - 1015

6 International Commission on Radiological Protection (ICRP). The 1990 Recommendations of the International Commission on Radiological Protection. ICRP Publication 60. Annals of the ICRP. Oxford: Pergamon Press; 1991

7 European guidelines on quality criteria for diagnostic radiographic images in paediatrics.. European Commission 1996, EUR 16261 EN

8 Noßke D. Bekanntmachung der aktualisierten diagnostischen Referenzwerte für diagnostische und interventionelle Röntgenuntersuchungen. Bundesamt für Strahlenschutz 22.6.2010

9 Born M, Spiller L, Bachour H et al. Das MCU bei Kindern. Dosiswerte im klinischen Alltag im Hinblick auf die stark gesenkten diagnostischen Referenzwerte. Fortschr Röntgenstr 2013; 185: 262-267

10 Hammer GP, Seidenbusch MC, Schneider K et al. Inzidenz von Kinderkrebs nach Röntgendiagnostik im Patientenkollektiv der Jahre 19762003 einer Universitäts-Kinderklinik. Fortschr Röntgenstr 2010; 182: 404-414 